ARTICLE

Received 30 Aug 2013 | Accepted 6 Feb 2014 | Published 4 Mar 2014

DOI: $10.1038 /$ ncomms 4399

OPEN

\title{
microRNA input into a neural ultradian oscillator controls emergence and timing of alternative cell states
}

Marc Goodfellow ${ }^{1, \dagger}$, Nicholas E. Phillips ${ }^{1}$, Cerys Manning ${ }^{1}$, Tobias Galla $^{2} \&$ Nancy Papalopulu ${ }^{1}$

Progenitor maintenance, timed differentiation and the potential to enter quiescence are three fundamental processes that underlie the development of any organ system. In the nervous system, progenitor cells show short-period oscillations in the expression of the transcriptional repressor Hes1, while neurons and quiescent progenitors show stable low and high levels of Hes1, respectively. Here we use experimental data to develop a mathematical model of the double-negative interaction between Hes1 and a microRNA, miR-9, with the aim of understanding how cells transition from one state to another. We show that the input of miR-9 into the Hes1 oscillator tunes its oscillatory dynamics, and endows the system with bistability and the ability to measure time to differentiation. Our results suggest that a relatively simple and widespread network of cross-repressive interactions provides a unifying framework for progenitor maintenance, the timing of differentiation and the emergence of alternative cell states.

\footnotetext{
${ }^{1}$ Faculty of Life Sciences, Michael Smith Building, The University of Manchester, Oxford Road, Manchester M13 9PT, UK. ${ }^{2}$ Theoretical Physics, School of Physics and Astronomy, The University of Manchester, Manchester M13 9PL, UK. †Present address: College of Engineering, Mathematics and Physical Sciences, University of Exeter, Exeter, Devon EX4 4QF, UK. Correspondence and requests for materials should be addressed to N.P. (email: nancy.papalopulu@manchester.ac.uk).
} 
$\mathrm{T}$ he nervous system is built by progenitor cells that divide either symmetrically or asymmetrically to give rise to neurons and glia ${ }^{1,2}$. Differentiated cells appear in a timed fashion, and although the timing of generation of different cell types is not as stereotypical as previously thought ${ }^{3}$, timing of differentiation is an important element in the correct development of the nervous system ${ }^{4}$. Some progenitors also need to replenish their population by symmetric proliferative or asymmetric divisions, whereby at least one of the daughter cells remains a progenitor. Such maintenance is imperative to avoid the premature depletion of the progenitor pool and to allow the nervous system to reach its final size. In addition, progenitors that remain at the end of neurogenesis may be transformed into quiescent progenitors that are able to generate neurons in the adult and be re-activated upon injury or neuronal $\operatorname{loss}^{1}$. Thus, the timing of differentiation, progenitor maintenance and the adoption of alternative cell states (that is, differentiation or quiescence) are three fundamental principles that underlie the development of the nervous system.

Experimental studies over several decades have elucidated some of the key molecular regulators of these processes. For example, Hes1, a basic helix-loop-helix transcriptional repressor activated in response to Notch signalling, has been shown to modulate the progenitor state. Hes1 knockout mice show premature neuronal differentiation accompanied by early progenitor depletion, while Hes1 overexpression prevents neuronal differentiation ${ }^{5,6}$. However, it is unclear how progenitor maintenance is co-ordinated with the timing of differentiation, the acquisition of alternative cells states and whether there is an underlying unifying mechanism for these processes.

The development of advanced live imaging techniques has allowed some hypotheses to be formulated in attempt to answer these questions. For example, the expression of Hesl has been shown to be much more dynamic than previously thought, displaying short-period (ultradian) oscillations in progenitor cells of different tissues ${ }^{7-10}$. Thus, based on a combination of experimental data and imaging of expression dynamics in normal development, it has been proposed that the Hes1 oscillatory state is necessary for the maintenance of progenitors, whereas sustained low or high levels are associated with differentiation or quiescence, respectively ${ }^{6}$. This hypothesis led us to suggest that understanding the mechanisms by which cells transition from the oscillatory state into sustained high or low levels of Hes1 may provide the long-sought unifying mechanism for co-ordination of the basic neurogenic processes.

Oscillations are predominantly caused by Hes1 autorepression, coupled with messenger RNA (mRNA) and protein instability ${ }^{11-13}$. Although mRNA instability is an essential component of oscillatory dynamics, the mechanisms of its regulation were unknown and therefore, mRNA degradation rate parameters were fixed in early models (with the exception of ref. 14). It was subsequently shown by us, and others, that miR-9 regulates the stability of Hes 1 mRNA and related genes in several model systems ${ }^{9,15-18}$ and is transcriptionally repressed by Hes 1 in the mouse ${ }^{9}$ or Her6 in zebrafish ${ }^{17}$. We hypothesized that the slow degradation rate of mature miR-9 can increase the degradation of Hes 1 mRNA over time, leading to an exit from oscillations. Thus, we suggested that the double-negative feedback loop of miR-9 and Hes1 provides a mechanism for the exit of oscillations with an embedded selflimited timer'.

Here we use mathematical modelling to analyse the potential of this simple, but fundamental, transcription factor/microRNA (miRNA) network to explain the aforementioned principles of nervous system development. The mathematical model incorporates the effects of miRNAs on both mRNA stability and translational repression, using recent experimental results to constrain its parameters. We then demonstrate that Hes $1 /$ miR-9 interactions can lead to high or low Hes1 levels depending upon the strength of repressive interactions. Furthermore, these levels can be reached by transient oscillations, the lengths of which represent the amount of time that a cell spends in the progenitor state. Finally, we demonstrate that initial concentrations of miR-9 anticipates the future cell state choice, in terms of differentiation or quiescence, defined by low or high stable Hes1 levels. This is due to the emergence of bistability in the model, which is brought about by the introduction of miR-9. Thus, a relatively simple network of mRNA-miRNA interactions is capable of shaping the timing and fate choice of neural progenitors. The provision of a cell autonomous but tuneable timing mechanism reconciles the influence of intrinsic and extrinsic factors that govern the timing of differentiation. Furthermore, our model provides a unifying framework for progenitor maintenance, the timing of differentiation and the acquisition of a differentiated state or quiescence, thus accounting for some of the fundamental principles of neurogenesis.

\section{Results}

Computational model of the Hes1-miR-9 interaction network. In Fig. 1, we provide a visual representation of the network of interactions between Hes1 and miR-9, based on experimental data ${ }^{9}$. In addition to the autorepression of Hes 1 transcription by Hes1 protein, the incorporation of miR-9 leads to three new network interactions, namely the repression of miR-9 production by Hes1 protein, miR-9-mediated changes in mRNA degradation rate and putative miR-9 repression of Hes1 protein translation ${ }^{9,18-20}$. We use delay differential equations to track the change in relative amounts of Hes1 protein $(p)$, mRNA $(m)$ and miR-9 $(r)$ over time arising due to 'production' and 'degradation' factors. The equations governing the dynamics of these three species are described sequentially below.

The influence of miR-9 on Hes 1 mRNA production. Equation (1) describes the temporal change of Hes1 mRNA concentration.

$$
\frac{\mathrm{d} m}{\mathrm{~d} t}=G(p(t-\tau))-S(r) m
$$

The autoinhibition of Hes1 acting as a repressor of transcription is implemented via the function $G$, the form of which is taken directly from previous models ${ }^{12-14}$ as follows:

$$
G(p(t-\tau))=\frac{1}{1+\left(\frac{p(t-\tau)}{p_{0}}\right)^{n_{0}}}
$$

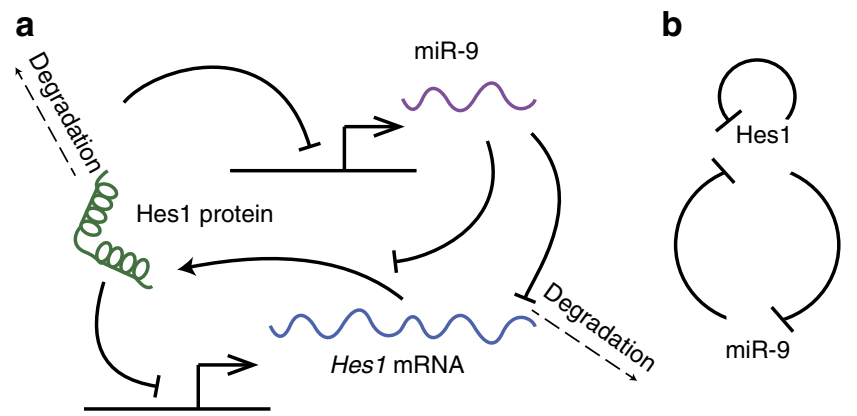

Figure 1 | Experimentally determined network interactions between Hes1 and miR-9. (a) Detailed visualization of the Hes1/miR-9 network. Solid arrows indicate production, whereas flat line ends represent repressive interactions. (b) Simplified network motif. 
As levels of $p(t-\tau)$ increase (where $t-\tau$ accounts for the delay in protein production due to transcription and translation), autoinhibition increases because $G$ decreases to small positive values and hence the total production term is reduced. The transition from minimal to maximal repression is governed by the shape of the function, $G$, which is determined by two parameters, $p_{0}$ and $n_{0}$. The parameter $p_{0}$ represents the amount of protein required to reduce Hes 1 transcription by half and therefore embodies the relative strength of repression: low values of $p_{0}$ imply that low values of protein can have a repressive effect, whereas high values for $p_{0}$ imply large amounts of protein are required for transcription to be attenuated (Supplementary Fig. 1). The parameter $n_{0}$ determines the steepness of $G$ and has previously been described as a Hill coefficient indicating, for example, the degree of co-operativity in the repressive interaction ${ }^{12}$. Here, we describe it as a more abstract term that simply alters the shape of this function, with higher values of $n_{0}$ making the transition from minimal to maximal repression more step-like, as shown in Supplementary Fig. 1b. In order to demonstrate the possible effects on the form of repression that arise from changes in $p_{0}$ and $n_{0}$, we provide examples in Supplementary Fig. 1.

The inclusion of miR-9 in the model means that the degradation term for Hes 1 mRNA $(m)$ is now a dynamic function of miR-9 $(r)$ levels. This function is informed by the experimental results of ref. 9, in which the authors measured the half-life of Hes 1 mRNA in wild-type cells as well as in cells injected with premiR-9 or containing Hes $13^{\prime}$ UTR with mutated binding sites, thus diminishing the effect of miR-9 (ref. 9). These results can be incorporated directly into our model since approximate bounds are provided for the half-life of Hes 1 mRNA when miR-9 is at high, low or intermediate levels. The results suggest that Hes1 mRNA half-life is bounded between $\sim 20 \mathrm{~min}$ for high levels of miR-9 and $35 \mathrm{~min}$ for low levels of miR-9, with the wild-type value in between these bounds. Taking this into account, we propose the following functional form for the role of miR-9 in altering Hes1 mRNA half-life:

$$
S(r)=b_{1}+\frac{b_{\mathrm{u}}-b_{\mathrm{l}}}{1+\left(\frac{r}{r_{0}}\right)^{m_{0}}}
$$

The general form of $S(r)$ is chosen to be equivalent to that of $G$ so that its shape is controlled by strength and shape parameters (here $r_{0}$ and $m_{0}$ ) (Supplementary Fig. 1). The parameters $b_{1}$ and $b_{\mathrm{u}}$ impose lower and upper bounds for Hes1 mRNA half-life and are fixed to $\ln (2) / 20 \mathrm{~min}^{-1}$ and $\ln (2) / 35 \mathrm{~min}^{-1}$, respectively.

The influence of miR-9 on Hesl protein production. Equation (4) describes the temporal change of Hes1 protein $(p)$ levels.

$$
\frac{\mathrm{d} p}{\mathrm{~d} t}=F(r) m-\frac{\ln (2)}{\mu_{p}} p
$$

We have incorporated a new function, $F(r)$, to capture the attenuation of protein production by miR-9. We retain the general form of $G$ so that the strength and shape of translational repression (here governed by the parameters $r_{1}$ and $m_{1}$, respectively) can be tuned as follows:

$$
F(r)=\frac{1}{1+\left(\frac{r}{r_{1}}\right)^{m_{1}}}
$$

These equations allow us to study how the presence of miR-9 affects the amount of Hes1 protein by examining its effect on mRNA degradation independently from its effects on translation repression (by setting $F(r)=1$ ). Doing so renders Hes1 protein production independent of translational repression by miR-9.
Taken together, equations (1) and (4) represent a description of the Hes1 oscillator in which the effect of miR-9 levels on its dynamics can be explored by treating the levels of miRNA, $r$, as a parameter. By setting $r$ to certain values and evaluating the dynamics of this reduced system (henceforth referred to as the 'two-variable' system; see below) one can generate a snapshot view of the way in which $r$ can affect the Hes1 oscillator. However, this view of the system neglects the feedback from $p$ onto $r$, hence a third equation tracking the dynamics of $r$ was developed.

The dynamics of miR-9 production. We introduce a new variable representing the levels of miR-9, $r$, and track its dynamics, taking into account the repression of miR- 9 transcription by Hes 1 as follows:

$$
\frac{\mathrm{d} r}{\mathrm{~d} t}=G_{r}(p(t-\tau))-\frac{\ln (2)}{\mu_{r}} r
$$

In equation (6), we assume that a constant upstream activation of miR-9 is attenuated due to the repressive activity of Hes 1 protein. This repression is provided by the function $G_{r}$, which follows the same general form as $G$. We allow for the case that repression by Hes 1 acts differently at the miR- 9 and Hes 1 loci by introducing different parameters for the strength and shape of repression in $G_{r}$, that is, $p_{1}$ and $n_{1}$ as follows:

$$
G_{r}(p(t-\tau))=\frac{1}{1+\left(\frac{p(t-\tau)}{p_{1}}\right)^{n_{1}}}
$$

Note that $G_{r}$ is shaped by $p_{1}$ and $n_{1}$ in the same way that $G$ is shaped by $p_{0}$ and $n_{0}$. Setting $\mu_{r}$ (the half-life of miR-9) to high values replicates the high stability of miR-9 observed experimentally 9 .

Degradation terms in the model are expressed as half-lives by introducing the factor $\ln (2)$. The parameters $\mu_{p}$ and $\mu_{r}$ are therefore the half-lives of Hes 1 protein and miR-9, respectively. The half-life of Hes 1 mRNA is affected by miR-9, and hence is given in the model by the function $\ln (2) / S(r)$.

In summary, the equations governing the system of Hes1 and miR-9 interactions are as follows:

$$
\begin{gathered}
\frac{\mathrm{d} m}{\mathrm{~d} t}=G(p(t-\tau))-S(r) m \\
\frac{\mathrm{d} p}{\mathrm{~d} t}=F(r) m-\frac{\ln (2)}{\mu_{p}} p \\
\frac{\mathrm{d} r}{\mathrm{~d} t}=G_{r}(p(t-\tau))-\frac{\ln (2)}{\mu_{r}} r
\end{gathered}
$$

The system described by equations (8) and (9) is referred to as the two-variable model, while equations $(8-10)$ are referred to as the full model.

Model assumptions. A full list of parameters and their default values is given in Table 1 , and a summary of the equations is given in Supplementary Note 1. The abstract formulation of the system we employ is based upon the following assumptions. We neglect the dynamics of external activating factors and focus instead on the internal dynamics of the Hes1/miR-9 system under the assumption that Hes 1 and miR-9 transcription are constitutively active when not repressed. Note that these activation factors are absorbed into the strength parameters of the repression functions ${ }^{12}$. To support our focus on intracellular mechanisms, we use bioluminescence imaging to demonstrate the persistence of oscillations in sparsely plated c17.2 neural progenitor cells in Supplementary Fig. 2. 
The dynamics of free, mature miR-9 are lumped into a single variable, $r$, thus neglecting the pre- and mature versions of this species. We therefore do not account for differential stability of pre- and mature miR-9 or the time delay in the production of the active molecule. Degradation of free miR-9, together with association and dissociation of the miR-9/Hes 1 complex, are lumped into the degradation term for $r$. Stability of free miR-9 is

\begin{tabular}{|c|c|c|c|}
\hline $\begin{array}{l}\text { Para- } \\
\text { meter }\end{array}$ & $\begin{array}{l}\text { Default } \\
\text { value }\end{array}$ & Interpretation & Reference \\
\hline $\bar{\tau}$ & $29 \min$ & $\begin{array}{l}\text { Time delay in Hes1 protein } \\
\text { production. }\end{array}$ & 21 \\
\hline$p_{0}$ & 390 & $\begin{array}{l}\text { Amount of protein required to reduce } \\
\text { Hes } 1 \text { mRNA transcription by half. }\end{array}$ & \\
\hline$n_{0}$ & 5 & Quantifies the step-like nature of $G$. & 12 \\
\hline$r_{0}$ & 100 & $\begin{array}{l}\text { Amount of miR-9 required to reduce } \\
\text { Hes } 1 \text { mRNA degradation rate by half. }\end{array}$ & \\
\hline$m_{0}$ & 5 & Quantifies the step-like nature of $S$. & \\
\hline$b_{1}$ & $\begin{array}{c}\ln (2) / \\
20 \min ^{-1}\end{array}$ & Lower bound for Hes1 mRNA half-life. & 9 \\
\hline$b_{\mathrm{u}}$ & $\begin{array}{l}\ln (2) / \\
35 \min ^{-1}\end{array}$ & Upper bound for Hes1 mRNA half-life & 9 \\
\hline$r_{1}$ & 300 & $\begin{array}{l}\text { Amount of miR-9 required to reduce } \\
\text { Hes1 protein translation rate by half. }\end{array}$ & \\
\hline$m_{1}$ & 5 & Quantifies the step-like nature of $F$. & \\
\hline$p_{1}$ & Varies & $\begin{array}{l}\text { Amount of protein required to reduce } \\
\text { miR-9 production rate by half. }\end{array}$ & \\
\hline$n_{1}$ & 5 & Quantifies the step-like nature of $G_{r}$ & \\
\hline$\mu_{r}$ & $1,000 \mathrm{~min}$ & Lumped half-life for free miR-9. & \\
\hline$\mu_{p}$ & $22 \mathrm{~min}$ & Half-life of Hes1 protein. & 11 \\
\hline
\end{tabular}

kept high by assuming that the net effect of this degradation is small (that is, $\mu_{r}$ is assumed to take large values). Four inhibitory interactions are modelled by decreasing functions of substrate ( $p$ or $r$ ); (i) Hes1 protein inhibiting Hes1 mRNA (G) (ii) Hes1 protein inhibiting miR-9 transcription $\left(G_{r}\right)$, (iii) miR-9 inhibiting Hes 1 mRNA transcription ( $S$ ) and (iv) miR-9 inhibiting Hes1 translation $(F)$. In the absence of specific information regarding the shape of these functions, we assume that they are governed by a function comprising parameters that control the shape $\left(n_{0}, n_{1}\right.$, $\left.m_{0}, m_{1}\right)$ and strength $\left(p_{0}, p_{1}, r_{0}, r_{1}\right)$ of inhibition.

Model oscillations occur for experimental mRNA half-lives. Experimental results suggest that Hes1 protein oscillations should exist for half-lives of Hes1 mRNA within the bounds of $\sim 20$ and $35 \mathrm{~min}$. At values more extreme than these, oscillations are not sustained $^{9,18}$. To discover whether our mathematical model recapitulates this experimental observation, we examined the presence of oscillations due to changes in the mRNA half-life, given by $S(r)$. We focused initially on the case in which miR-9 acts only to reduce the stability of Hes $1 \mathrm{mRNA}^{9}$, by setting $F(r)=1$ to negate the effects of $r$ on translation of Hes1 protein $(p)$ in the two-variable model (equations (8) and (9)).

The parameter that defines the steepness of Hes 1 transcriptional autorepression, $n_{0}$, was fixed to a value that has been shown to generate oscillations (that is, $n_{0}=5$ (ref. 12), but the relative strength of transcriptional autorepression by Hes1 protein, $p_{0}$, and the time delay in protein production, $\tau$, were varied as shown in Fig. 2. The delay, $\tau$, was allowed to take values in the physiologically plausible range of $20-30 \mathrm{~min}^{21}$. Figure 2 shows that, for each value of $p_{0}$, oscillations exist as the half-life of Hes 1 mRNA $(\ln (2) / S(r))$ is decreased. Furthermore, when $\tau=29 \mathrm{~min}$, a window of oscillations arises that lies within the experimentally
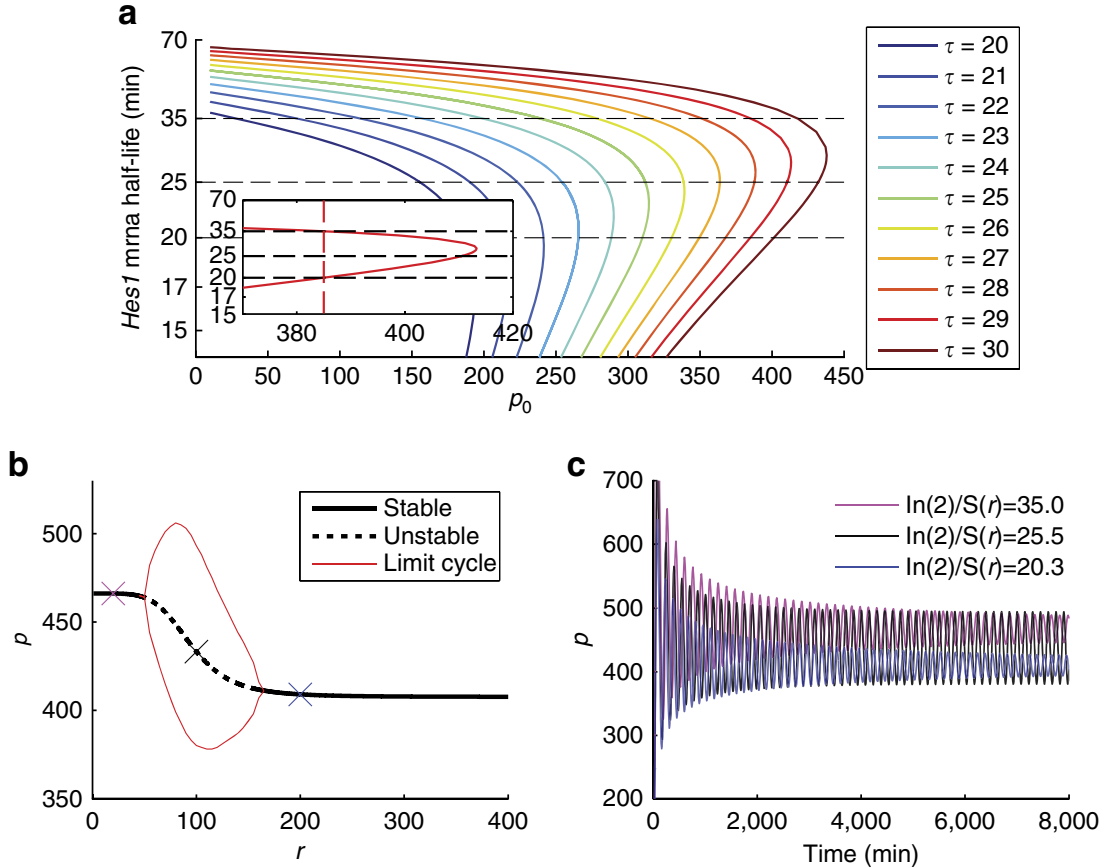

Figure 2 | Presence of oscillations in the model when miR-9 acts only to affect the stability of Hes1 mRNA. (a) Different combinations of $\tau, p_{0}$ and $S(r)$ are used to test for the presence of oscillations. The different coloured lines denote Hopf bifurcations for different values of $\tau$ as indicated. A Hopf bifurcation denotes the transition of a system from a stable to an unstable, oscillatory state or vice versa, as a parameter of the system is varied. Fixed points exist to the right of the curves whereas oscillations are present to the left. The inset shows the curve for $\tau=29$. The dashed red line indicates the value of $p_{0}$ for which two Hopf bifurcations exist for mRNA half-lives of $\sim 35$ and 20 min. (b) A window of oscillations emerges for changes in $r$, with $p_{0}$ fixed at 390 and $\tau=29 \mathrm{~min}$. (c) Example time series when $r$ is fixed to the values given by crosses in (b). These $r$ values give rise to the half-lives (In(2)/ $S(r))$ as indicated. $n_{0}=5, \mu_{p}=22 \mathrm{~min}, b_{\mathrm{I}}=\ln (2) / 20 \mathrm{~min}^{-1}, b_{\mathrm{u}}=\ln (2) / 35 \mathrm{~min}^{-1}, r_{0}=100, m_{0}=5$. 
determined values of mRNA stability when $p_{0}$ is around 390 (arbitrary units) (inset of Fig. 2a,b). Example time series are shown in Fig. 2c. In contrast to the sustained oscillations when Hes 1 mRNA half-life is around wild-type levels $(\ln (2) /$ $S(r)=27 \mathrm{~min}$ (ref. 9)), oscillations dampen for parameters chosen outside the half-life boundaries reported in ref. 9 .

Although the effect of miR-9 on Hes1 mRNA stability has been experimentally demonstrated, it is increasingly clear that miRNAs exert a combined effect on mRNA stability and translation ${ }^{22-24}$. To account for a combined action on Hes1 mRNA degradation and translational repression, we introduced $F(r)$ into the two-variable model. In this scenario, as $r$ is varied over increasing positive values, the two repressive effects of $r$, that is, the degradation of Hesl mRNA and inhibition of the translation of Hes1 protein, act simultaneously. Supplementary Fig. 3 demonstrates that several parameter sets allow the generation of sustained oscillations under these circumstances. Furthermore, Fig. 3a,b demonstrate that an oscillatory window that contains the wild-type mRNA half-life value exists, in line with the results of Bonev et al. ${ }^{9}$, when the strength of the translational repression is relatively small (large $r_{1}$ ) and the steepness of the repressive interactions relatively high $\left(m_{0}=m_{1}=5\right)$. These results show that our model recapitulates the experimentally observed window of oscillations ${ }^{9}$ either by mRNA degradation acting alone or in combination with translational repression.

In summary, we have demonstrated that the experimentally observed window of oscillations can be present in the model for relevant values of the Hes 1 mRNA degradation rate. In the remainder of the paper, we investigate the effects of incorporating miR-9 interactions into the network. We fix $\tau, p_{0}, m_{0}, m_{1}, r_{0}$ and $r_{1}$ to the values given in Table 1, which represents a set of values for which the alignment with experiments holds. Other possible parameter values can be used, as demonstrated in Figs 2,3 and Supplementary Fig. 3 but these would not necessarily allow the model to match experimental observations.

Attaining low values of Hes1. Experimental evidence shows that differentiated neurons are characterized by low stable levels of
Hes1 protein ${ }^{25}$. We were therefore particularly interested in defining the conditions under which this dynamic network achieves low stable levels of Hes1. We analysed the two-variable model and focused initially on the case that miR-9 acts only to reduce the stability of Hes1 mRNA by setting $F(r)$ to 1 , thus removing the influence of miRNA on the protein production rate. The stability and steady-state values of Hes1 protein were calculated and are shown in Fig. 2b. It can be seen that the system displays an oscillatory window (unstable dynamics, or limit cycle') while the final protein levels on either side of the oscillatory window are stable, either high or low. However, the accumulation of miR-9 can reduce the levels of Hes1 protein only by $\sim 12 \%$ due to its action on mRNA degradation alone (Fig. $2 b$ ). A difference in concentration of this magnitude has been shown to be capable of mediating different cell states in the context of morphogens in Drosophila embryos ${ }^{26}$. However, neurons express very low or no levels of Hes1 (refs 25,27); therefore, it is unclear whether such a small change would be sufficient in the developing nervous system. We therefore sought to determine the conditions that allow for a greater degree of attenuation of Hes 1 protein, by allowing miR-9 to repress Hes1 translation (that is, by allowing $F(r)$ to vary). In this case, since $F(r)$ can take values close to zero, the steady-state value of $p$ can also, in principle, reach values close to zero. Thus, with translation repression acting alongside mRNA degradation, miR-9 is able to reduce Hes1 to lower stable state levels than when miR-9 acts solely to increase the degradation rate of Hes1 mRNA (Fig. 3c, compare with Fig. 2b). The exact steady-state value of $p$ attained depends upon all of the parameters of the system. We henceforth focus on the case that $p$ is close to zero at high levels of $r$, though we note that a continuum of steady-state values of $p$ can be attained by adjusting the parameters of the system.

Including miR-9 in the Hes1 oscillator leads to bistability. Progenitors give rise to differentiated neurons but may also become quiescent, or slowly proliferating cells. Slowly proliferating, non-neurogenic progenitors, are found in boundary
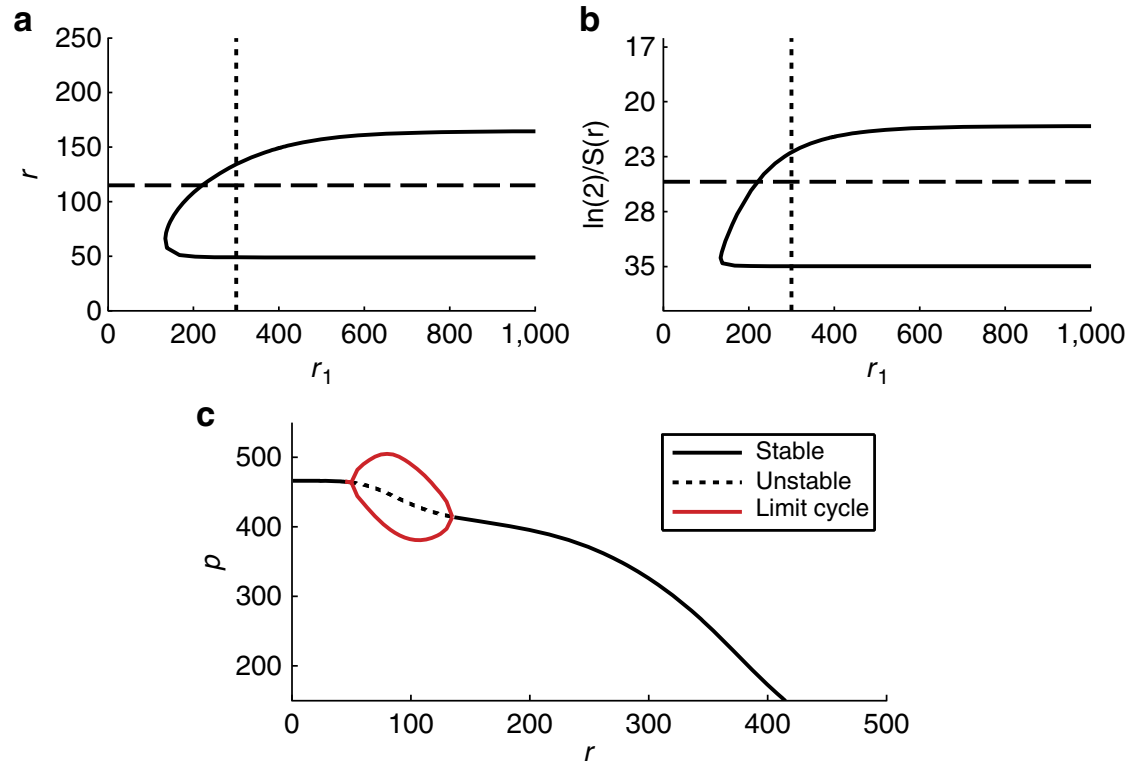

Figure 3 | Presence of oscillations in the two-variable model when $\boldsymbol{r}$ acts via both mRNA degradation and translational repression. (a) Shows the location of Hopf bifurcations as $r_{1}$ and $r$ are varied. Here, oscillations exist to the right of the curve. The horizontal line indicates the value of $r$ for which $\ln (2) / S(r)=25$. The vertical dashed line indicates a value of $r_{1}=300$. (b) as (a) but with y axis plotted in terms of mRNA half-life ( $\ln (2) / S(r)$ ). (c) A window of oscillations emerges for changes in $r$. The addition of translational repression allows a lower steady state for high $r$. Other parameters are $p_{0}=390, \tau=29 \mathrm{~min}, b_{1}=\ln (2) / 20 \mathrm{~min}^{-1}, b_{\mathrm{u}}=\ln (2) / 35 \mathrm{~min}^{-1}, n_{0}=m_{0}=m_{1}=5, r_{0}=100, \mu_{p}=22 \mathrm{~min}$. 
regions of the central nervous system and they are characterized by high stable levels of Hes1 (ref. 25). Progenitors may also enter a quiescent or slowly dividing state at the end of embryonic neurogenesis ${ }^{1,28}$. We therefore asked whether our model can lead to a mechanistic understanding of how this cell state is attained. Comparing the levels of $p$ at either side of the oscillatory window in Fig. 3c demonstrates that higher steady-state levels of Hes1 protein are obtained when miR-9 levels are low (and hence Hes 1 mRNA half-life longer). However, this analysis does not take into account the mechanisms by which different miR-9 levels are attained. The findings of ref. 9 highlight that miR-9 is a dynamic component of the network and, therefore, we introduced $r$ as a dynamic variable in the model. We assume that the production of miR-9 is constitutive but repressed by Hes1 (ref. 9) as described in equation (10) of the full Hes1/miR-9 network. The sum of the production and degradation rates determine whether miR-9 levels increase or decrease over time.

Figure $4 \mathrm{a}$ shows a bifurcation diagram for changes in the strength of repression of miR-9 by Hes $1\left(p_{1}\right)$ in the full model. It can be seen that there are two branches of stable fixed points, which represent the high and low stable values of Hes1 described earlier. These states are produced in the model in an intuitive way due to changes in $p_{1}$. When the strength of repression of miR-9 by Hes 1 is low (high $p_{1}$ ), miR-9 accumulates due to weak repression by Hes1 and this leads to a stable state with low Hes1 $(p)$. However, as the strength of repression of miR-9 by Hes 1 increases (decreasing $p_{1}$ ), a new stable steady state emerges that has high Hes1 levels.

Figure $4 \mathrm{a}$ also shows that the network interactions of the full model give rise to a state of bistability between the high and low Hes1 states. Bistability (here due to fold bifurcations) means that, for identical parameter settings, the model can attain either sustained high or sustained low levels of Hes1 depending upon the levels of $m, p$ and $r$ that it is initiated with. Example time series for a bistable situation are shown in Fig. 4b,c. Figure $4 \mathrm{~b}$ demonstrates that initially high levels of miR-9 $(r)$ accumulate and maintain the suppression of Hes1 $(p)$. In contrast, initially low levels of miR-9 $(r)$ remain low, and lead to higher, stable levels of Hes1.

A Hopf bifurcation that gives rise to stable oscillations resides on the top branch of fixed points, and therefore the high Hes1 steady state is a focus near this bifurcation. A consequence of this is that the steady state on the top branch is 'excitable' in that it can produce transient oscillations in response to a brief perturbation to the system. An example of this excitability is shown in Fig. 5a, wherein a small, immediate increase in $m$ can cause the onset of transient oscillations. In contrast, the steady state on the lower branch does not produce transient oscillations in response to this perturbation, or other, much larger instantaneous changes in $m$ (data not shown). We sought an alternative means by which transient oscillations could be initiated from the low Hes1 steady state, and found that a prolonged, pulse perturbation to $p$ could achieve this aim, as demonstrated in Fig. 5b. This difference in stability of the top and bottom branches is consistent with their representation of quiescent progenitors and differentiated neurons, respectively. Our interpretation of the result in Fig. 5 is that quiescent progenitors (high steady-state Hes1) are readily re-activated, consistent with the current thinking that they are 'poised for activation $^{29}$ whereas the differentiated state is robust to change.

Timing transitions from oscillatory to stable Hes1 dynamics. Having shown that high or low steady-state levels of Hes1 can be attained, and even exist in a bistable regime, it is important to consider how the length of time a cell spends as a progenitor
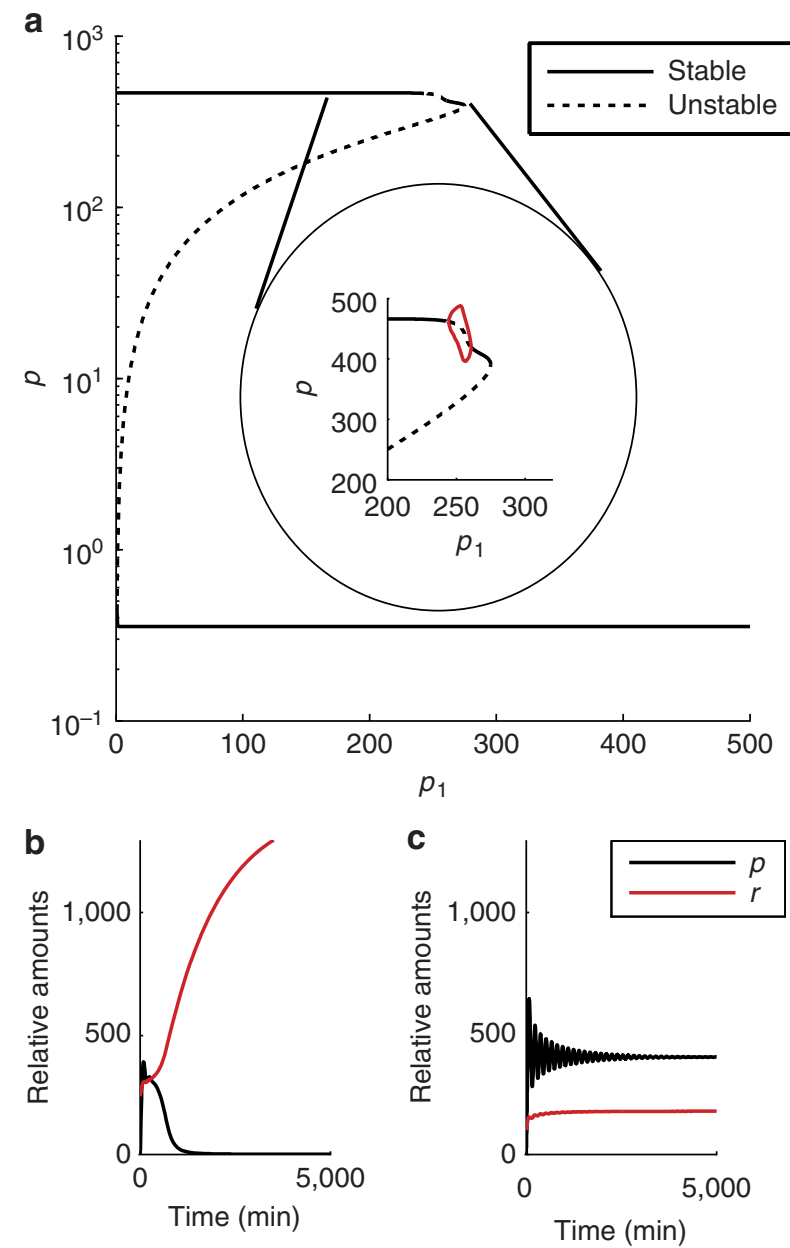

Figure 4 | Oscillations and different steady-state levels of Hes1 in the full system for changes in the strength of repression of miR-9 by Hes1 $\left(\boldsymbol{p}_{1}\right)$. (a) Shows a bifurcation diagram for changes in $p_{1}$, with protein levels ( $p$ ) given as output on the $y$ axis. The circled area shows a magnified region of parameter space that contains oscillations (limit cycle maxima and minima given by red lines). (b,c) Show example of time series for a case of bistability $\left(p_{1}=272\right)$. The initial conditions (giving a constant history vector) are (b) $m(0)=0, p(0)=0, r(0)=240$ and (c) $m(0)=0, p(0)=0$, $r(0)=100$. Other parameters are $p_{0}=390, \tau=29 \mathrm{~min}, b_{1}=\ln (2) /$ $20 \min ^{-1}, b_{u}=\ln (2) / 35 \mathrm{~min}^{-1}, n_{0}=n_{1}=m_{0}=m_{1}=5, r_{0}=100, r_{1}=300$, $\mu_{p}=22 \mathrm{~min}, \mu_{r}=1,000 \mathrm{~min}$.

before activating differentiation pathways is controlled. We define the length of time that a cell remains as an actively proliferating progenitor as the length of time that Hes1 shows oscillatory dynamics. Introducing miR-9 as a dynamic variable in the system allows miR-9 to increase over time when the production term exceeds the degradation term, recapitulating the increase that is observed experimentally under proliferating conditions. Figure 6 demonstrates two possible ways by which different durations of oscillations can be achieved, before reaching a stable state with low levels of Hes1.

The first of these is a change of parameters. Figure 6a shows that increasing the strength of repression of miR-9 by Hes 1 (decreasing $p_{1}$ ) leads to longer transient oscillations. The reason for the lengthening of the transient is that the build up of miR-9 is initially slower, such that the threshold of miR-9 at which oscillations no longer exist is reached at a later time point.

A second way by which the timing of exit from oscillations can be controlled is by the initial amount of miR-9 present in the 
system, as demonstrated in Fig. 6b. When $r$ is initially high, Hes1 is more rapidly repressed to the non-oscillatory state. This is because $r$ resides closer to, or even beyond the threshold at which it can be substantially repressed by Hes1 protein and therefore levels of $r$ more rapidly increase, further suppressing Hes1.

The transient oscillations of Fig. 6 dampen in amplitude over time. It is therefore important to consider whether the corresponding oscillations in cultured cells would be detectable by our experimental methods. In order to investigate this point,
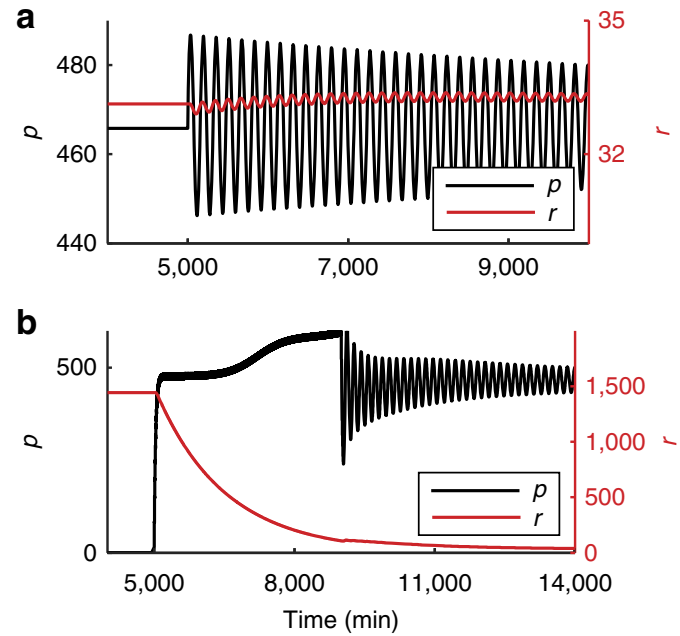

Figure 5 | The high Hes1 steady state can be readily excited into transient oscillations. (a) The model is initiated in the high Hes1 state in a bistable regime (parameters as default and $p_{1}=220$, initial conditions $m=14.6766, p=465.8128, r=33.1285)$. At 5,000 min, the Hes1 mRNA levels are instantaneously set to a value $10 \%$ higher than at this steady state (that is, $m=16.1433$ ). The system is excited into transient oscillations. (b) The model is initiated in the low Hes1 steady state (initial conditions $m=28.9, p=0.4, r=1442.6$ ). To excite the system, at $t=5,000 \mathrm{~min}$, a perturbation of 15 units of protein (an initial 30 -fold increase) is added every minute for $4,000 \mathrm{~min}$. Other parameters are $p_{0}=390, \tau=29 \mathrm{~min}$, $b_{1}=\ln (2) / 20 \mathrm{~min}^{-1}, b_{\mathrm{u}}=\ln (2) / 35 \mathrm{~min}^{-1}, n_{0}=n_{1}=m_{0}=m_{1}=5, r_{0}=100$, $r_{1}=300, \mu_{p}=22 \mathrm{~min}, \mu_{r}=1,000 \mathrm{~min}$.
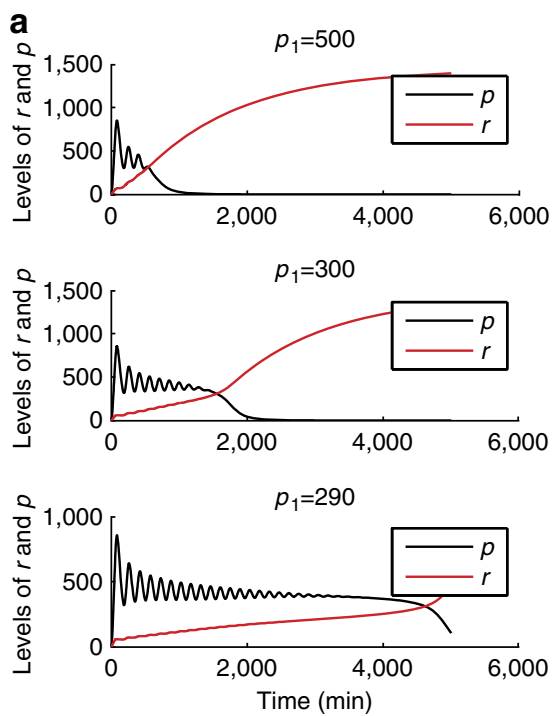

Figure 6 | Different durations of oscillations can be found for changing $\boldsymbol{p}_{\mathbf{1}}$ or the initial levels of $\boldsymbol{r}$. (a) Shows simulations with fixed $r(0)=0$ but changing $p_{1}$. (b) Shows simulations with fixed $p_{1}=290$ but changing $r(0)$. Here, $m(0)=0$ and $p(0)=0$. Other parameters are $p_{0}=390, \tau=29$ min, $b_{1}=\ln (2) / 20 \mathrm{~min}^{-1}, b_{\mathrm{u}}=\ln (2) / 35 \mathrm{~min}^{-1}, n_{0}=n_{1}=m_{0}=m_{1}=5, r_{0}=100, r_{1}=300, \mu_{p}=22 \mathrm{~min}, \mu_{r}=1,000 \mathrm{~min}$. b
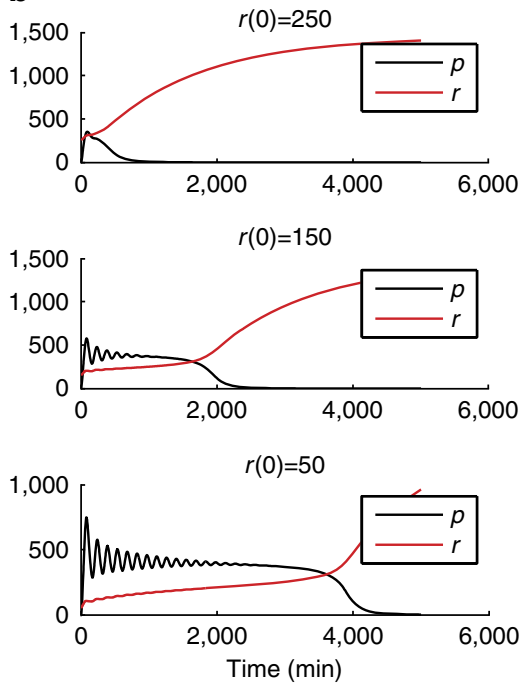

we measured Hes1 expression using bioluminescence imaging of c17.2 cells stably transfected with a Hes1::ub-luciferase reporter. We extracted peaks in an objective way and quantified their amplitude and period. Supplementary Fig. 4 demonstrates that the amplitude and period of the model oscillations are within the range of experimentally quantified values. In the case of dampening oscillations in the example of Supplementary Fig. 4, only the last 3 cycles out of 10 are close to the lower bound of amplitudes that were detected in c17.2 cells, and therefore the majority of these model oscillations would, in principle, be detected experimentally.

A comprehensive analysis of the presence of Hopf and fold bifurcations in the full system and its resulting dynamics is shown in Fig. 7 and Supplementary Fig. 5. Hopf and fold bifurcations exist for a range of $n_{1}, p_{1}$ and $\mu_{r}$ values, although their relative position in parameter space is dependent on the choice of $n_{1}$.

\section{Discussion}

In this paper, we used mathematical modelling to demonstrate that a negative feedback circuit between miRNA and an epressive transcription factor can unify the basic principles of neurogenesis; progenitor maintenance, acquisition of a differentiated state or quiescence and the timing of differentia. Our model incorporates experimentally constrained paraprovides a unifying theory for cell fate progression, which is likely to be applicable to any organ system that develops from dividing progenitor cells.

Our principal finding is that the amount of active miR-9 available in the cell provides a mechanism to tune the degradation rate of Hes 1 mRNA. From then on, we show that the introduction of miR-9 as a dynamic entity in the Hes1 oscillator has a profound effect on the properties of the regulatory network, over and above the Hes1 oscillator (shown comprehensively in Fig. 7).

First, it gives the Hes1 oscillator the ability to attain either high or low stable Hes1 states. In development, this could account for the ability of progenitor cells to progress either towards differentiation or towards a quiescent, slowly dividing state. Bistability in the system is due to the double-negative feedback loop 30,31 , which allows the network to settle into a high or low 

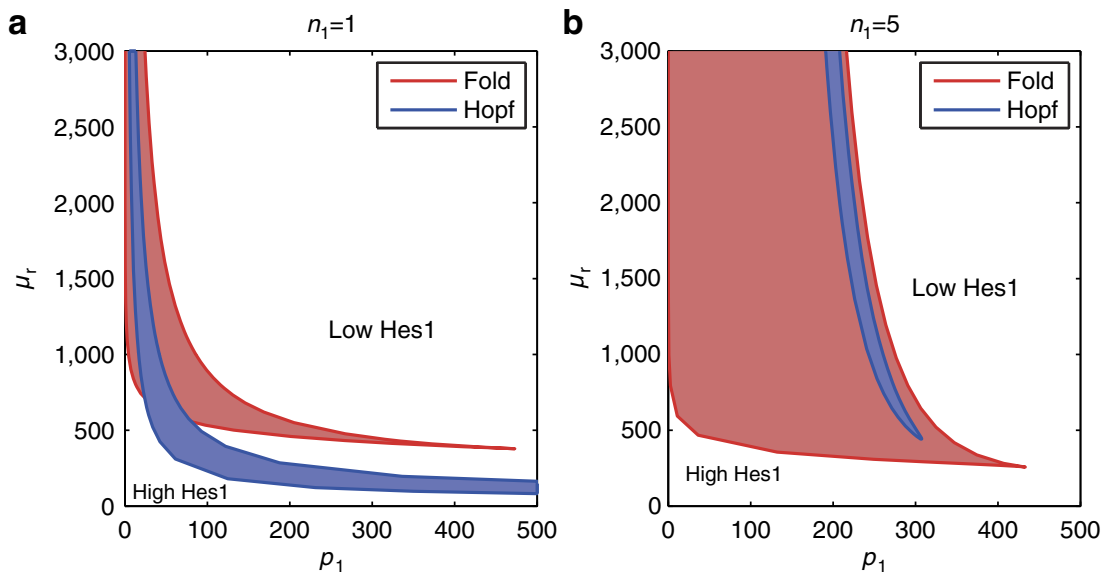

Figure 7 | The model can display bistability, sustained oscillations, stable high or low levels of Hes1 depending upon its parameterization. Local bifurcations are shown for changes in the miR-9 degradation rate, $\mu_{n}$ the strength of repression of miR-9 production by Hes 1 , $p_{1}$ and the shape of repression of miR-9 production by Hes1, $n_{1}$. The presence of Hopf and fold bifurcations are indicated by blue and red solid lines, respectively. Fold bifurcations lead to the creation or elimination of a pair of fixed points. Blue regions, therefore, indicate the presence of oscillations, while red regions indicate bistability. White regions represent the case of a single steady state with either high or low Hes1 levels (as indicated by the annotation). (a) $n_{1}=1$. (b) $n_{1}=5$. Other parameters are $p_{0}=390, \tau=29 \mathrm{~min}, b_{1}=\ln (2) / 20 \mathrm{~min}^{-1}, b_{\mathrm{u}}=\ln (2) / 35 \mathrm{~min}^{-1}, n_{0}=m_{0}=m_{1}=5, r_{0}=100, r_{1}=300, \mu_{p}=22 \mathrm{~min}$.

Hes1 stable state solely by adjusting the initial level of miR-9. Such bistability may account for the ambivalent state of progenitors reported in ref. 17. Alternatively, high or low Hes1 states can be attained by changing the parameters of the system, such as various forms of repression strength. Differential inheritance of miR-9 during mitosis can easily account for varying starting levels of miR-9 in daughter cells. Such differential inheritance could be either stochastic ${ }^{32}$ or the result of fate asymmetric divisions, which are prevalent in the development of the nervous system ${ }^{2}$.

Second, introducing miR-9 as a dynamic entity gives the network the ability to measure lapsed time. In turn, if the transition from oscillatory to stable dynamics marks the transition of cell states, it follows that the network has the ability to time the duration of progenitor maintenance, defined as the time that cells spend in the oscillatory state. We demonstrated that in the model, the timing of transient oscillations can be affected by intrinsic factors (the initial concentration of miR-9) or by factors extrinsic to the model (changes of parameters, see Fig. 6).

The measurement of biological time has been one of the most elusive areas of biology. While progress has been made on understanding cyclical time, found in biological clocks such as the circadian clock ${ }^{33}$, linear time progression has been more difficult to understand, perhaps with the exception of the temporal control of somite segmentation $^{34}$. Our model provides a mechanism by which cyclical time can be converted into linear time. Finally, the observation that the duration of oscillations can vary depending on the parameters of the system or the initial conditions means that although the timer is cell autonomous, it can be externally tuned, by extrinsic factors such as extracellular signalling or cell interactions.

This property endows cells with an innate ability to measure time, while having the flexibility to adjust the timer to external stimuli. We propose that this model reconciles the observation that timed differentiation can be cell autonomous ${ }^{35}$, or can be influenced by extrinsic factors, such as signals from the stem cell niche (reviewed in Okano and Temple ${ }^{36}$ ). In principle, such extrinsic factors could act in a number of ways, modifying one or more of the parameters of the network such as the strength of repressive interactions. A good example of this is the presence of co-factors (such as Id) that may diminish the autorepressive strength of Hes1 (ref. 37).

Double-negative feedback loops of the sort described here are extremely widespread in biology and particularly in developing systems. Such double-negative loops have been reported between transcription factors (see D-V patterning of the neural tube ${ }^{38}$ ) or even between transcription factors and miRNAs ${ }^{31,39}$. Thus, we predict that the network behaviour that we have described here is widely used by developing systems, the immune system and stem cells, where oscillatory gene expression is also observed ${ }^{40-42}$. Indeed, similar network motifs have been described that account for multi-state gene expression and oscillations due to spatial morphogen gradients ${ }^{43}$. Although the network topology is similar, the output of our model is temporal rather than spatial.

There are several questions that remain to be answered; our model serves to highlight and predict areas of high priority. In many cases, our numerical investigation of the model indicated a switch type behaviour in inhibitory interactions based on the assumption of a relatively steep transition from minimum to maximum repression. Biophysical measurements, such as the precise strength and shape of transcriptional and post-transcriptional repressive interactions and their effect on the duration of oscillations will need to be established in vivo in order to test the validity of such assumptions. Future experimentation should also establish quantitative measurements of the abundance of Hes 1 and miR-9 in progenitors and neurons, and examine whether there is differential inheritance during cell division that may predict the fate of daughter cells. Simultaneous imaging of dynamic gene expression and cell fate transition, coupled with optogenetic methods for manipulating oscillations (as recently described in ref. 10) will directly test some of the timing ideas put forward in this paper.

Overall, our data highlights miRNA processing and biogenesis as an important area of research ${ }^{44}$, since the output of our model is sensitive to the concentration of active miR-9. Wider adoption of live imaging techniques and quantitative methods for such highly dynamic gene expression will help the community provide answers to some of the emerging questions.

\section{Methods}

Experimental methods. c17.2 neural progenitor cells (Sigma-Aldrich) were grown in Dulbecco's Modified Eagle medium with $10 \%$ fetal bovine serum (FBS). To generate stable Hes1 reporter cell lines, pcDNA4-Hes1::ubq-luciferase WT 3'UTR was transfected into c17.2 cells using Lipofectamine 2000 (Invitrogen). Forty-eight hours after transfection, cells were plated into $1 \mathrm{mg} \mathrm{ml}^{-1}$ Zeocin (Invitrogen) and maintained in antibiotic selection for 2 weeks. Stable transfectants were plated at low density and individual resistant colonies picked to generate single-cell clones. 
c17.2 Hes1::ubq-luciferase clones were tested for luciferase expression and positive response to transient NICD overexpression on a FLUOstar Omega plate reader (BMG LABTECH). A representative clone was used for subsequent imaging. In bioluminescent imaging experiments, c17.2 Hes1::ubq-luciferase reporter cells were plated on $35 \mathrm{~mm}$ glass-based dishes (Greiner-Bio One) in sparse $(14,000$ cells per $\mathrm{ml})$ or confluent conditions ( 50,000 cells per $\mathrm{ml})$. Sparse cells were allowed to adhere before FBS withdrawal for $3 \mathrm{~h}$ and subsequent imaging in the presence of $10 \%$ FBS. Confluent cells were serum starved for $24 \mathrm{~h}$ before imaging in $10 \%$ FBS. $1 \mathrm{mM} \mathrm{D}$-luciferin (Promega) was added prior to imaging and plates were placed on an inverted microscope stage and maintained at $37^{\circ} \mathrm{C}$ in $5 \% \mathrm{CO} 2$. Luminescent images were obtained using a $10 \times 0.3 \mathrm{NA}$ air objective and collected with a cooled charge-coupled device camera (Orca II ER, Hamamatsu Photonics). A 30 -min exposure and $4 \times 4$ binning were used.

Bioluminescent movies were analysed using Imaris (Bitplane). Images were subject to smoothing using a $3 \times 3$ median filter. Individual cells and background regions were tracked manually using the 'spots' function. Single-cell bioluminescence values over time were extracted and subsequently analysed for the presence of peaks using MATLAB 7.12 (The MathWorks, Natick, MA, US) (see below).

Data analysis. We sought to define real peaks and troughs in the data that were distinct from background fluctuations (Supplementary Fig. 7a,b) and spurious changes in ongoing trends (Supplementary Fig. 7b,c).

The first step of the analysis was to calculate background measurements from the data. To this end four control regions of a plate, for each experiment, were identified by the absence of luminescent cells. Sequential changes in luminescence in control regions gave a distribution of values, and we required that real peaks had amplitudes greater than the majority of these. Thus, we set the value of the fluctuation at the 99th percentile in the distribution as a threshold above which the amplitude of real peaks should lie. This process is demonstrated in Supplementary Fig. 6 .

All turning point maxima in single-cell data were identified using the MATLAB function 'findpeaks', and it was assessed whether their amplitude was greater than the background threshold defined above. We defined amplitude as the change in luminescence measured from the peak to subsequent and previous time points (that is, we considered the descent on either side of the peak), greater than onetime step away. We defined a window length of 15 data points $(7.5 \mathrm{~h})$ either side of the peak, which allowed us to include peaks riding on slow changes in luminescence. Shorter windows neglected peaks with long period, whereas larger windows could generate spurious peaks due to chance drops in luminescence at distant time points. Supplementary Fig. 7a,b demonstrate the amplitude thresholding step.

An undesired consequence of the window approach is that peaks occurring as shoulders on larger peaks could still pass the amplitude threshold criterion. Therefore, we required that a significant drop in luminescence (using the same threshold as previously) occurred on either side of the peak before the next peak was encountered. An example of this final processing step is shown in Supplementary Fig. 7c. Troughs were identified by running the algorithm on the negative of the time series.

Having defined 'real' peaks and troughs in the data as above, we could proceed to calculate the amplitude and period of oscillations in bioluminescent data. The amplitude was defined as the percentage change in luminescence above background. The mean value over time of each four control regions was calculated, and the lowest of these values designated as a baseline. For each peak, its previous and subsequent troughs were identified and the baseline subtracted from each peak and trough. The two troughs either side of each peak gave two possible estimates for the peak amplitude. The normalized amplitude was defined as the minimum (over these two possible choices) ratio of baseline subtracted peak to trough ((peak-baseline)/(trough-baseline)). The normalized amplitude of the model peaks was calculated in the same way. In this case, the background was taken to be the eventual steady state of protein levels in the model. The period of oscillations in the model and in the data was calculated as the difference in time between each consecutive pair of peaks. All analyses and simulations were performed using MATLAB. For simulations of time series, we used the MATLAB function 'dde23' with options 'RelTol' $=10^{-5}$ and 'AbsTol' $=10^{-8}$. For history vectors, we used a single value repeated over prior time points. Numerical bifurcation analysis was performed using DDE-BIFTOOL ${ }^{45}$.

\section{References}

1. Malatesta, P., Appolloni, I. \& Calzolari, F. Radial glia and neural stem cells. Cell Tissue Res. 331, 165-178 (2008).

2. Götz, M. \& Huttner, W. B. The cell biology of neurogenesis. Nat. Rev. Mol. Cell Biol. 6, 777-788 (2005).

3. He, J. et al. How variable clones build an invariant retina. Neuron $\mathbf{7 5}, 786-798$ (2012).

4. Franco, S. J. \& Müller, U. Shaping our minds: stem and progenitor cell diversity in the mammalian neocortex. Neuron 77, 19-34 (2013).
5. Kageyama, R., Ohtsuka, T. \& Kobayashi, T. The Hes gene family: repressors and oscillators that orchestrate embryogenesis. Development 134, 1243-1251 (2007).

6. Kageyama, R., Ohtsuka, T., Shimojo, H. \& Imayoshi, I. Dynamic Notch signaling in neural progenitor cells and a revised view of lateral inhibition. Nat Neurosci. 11, 1247-1251 (2008).

7. Masamizu, Y. et al. Real-time imaging of the somite segmentation clock: revelation of unstable oscillators in the individual presomitic mesoderm cells. Proc. Natl Acad. Sci. USA 103, 1313-1318 (2006).

8. Shimojo, H., Ohtsuka, T. \& Kageyama, R. Oscillations in notch signaling regulate maintenance of neural progenitors. Neuron 58, 52-64 (2008).

9. Bonev, B., Stanley, P. \& Papalopulu, N. MicroRNA-9 modulates Hes1 ultradian oscillations by forming a double-negative feedback loop. Cell Rep. 2, 10-18 (2012).

10. Imayoshi, I. et al. Oscillatory control of factors determining multipotency and fate in mouse neural progenitors. Science 342, 1203-1208 (2013).

11. Hirata, H. et al. Oscillatory expression of the bHLH factor Hes1 regulated by a negative feedback loop. Science 298, 840-843 (2002).

12. Monk, N. Oscillatory expression of Hes1, p53, and NF- kappa B driven by transcriptional time delays. Curr. Biol. 13, 1409-1413 (2003).

13. Momiji, H. \& Monk, N. A. M. Dissecting the dynamics of the Hesl genetic oscillator. J. Theor. Biol. 254, 784-798 (2008).

14. Xie, Z.-R., Yang, H.-T., Liu, W.-C. \& Hwang, M.-J. The role of microRNA in the delayed negative feedback regulation of gene expression. Biochem. Biophys. Res. Commun. 358, 722-726 (2007).

15. Bonev, B., Pisco, A. \& Papalopulu, N. MicroRNA-9 reveals regional diversity of neural progenitors along the anterior-posterior axis. Dev. Cell 20, 19-32 (2011).

16. Leucht, C. et al. Microrna-9 directs late organizer activity of the midbrainhindbrain boundary. Nat. Neurosci. 11, 641-648 (2008).

17. Coolen, M., Thieffry, D., Drivenes, Ø., Becker, T. S. \& Bally-Cuif, L. mir-9 controls the timing of neurogenesis through the direct inhibition of antagonistic factors. Dev. Cell 22, 1052-1064 (2012).

18. Tan, S.-L., Ohtsuka, T., González, A. \& Kageyama, R. MicroRNA9 regulates neural stem cell differentiation by controlling Hes1 expression dynamics in the developing brain. Genes Cells 17, 952-961 (2012).

19. Pasquinelli, A. E. MicroRNAs and their targets: recognition, regulation and an emerging reciprocal relationship. Nat. Rev. Genet. 13, 271-282 (2012).

20. Filipowicz, W., Bhattacharyya, S. N. \& Sonenberg, N. Mechanisms of posttranscriptional regulation by microRNAs: are the answers in sight? Nat. Rev. Genet. 9, 102-114 (2008).

21. Lewis, J. Autoinhibition with transcriptional delay: a simple mechanism for the zebrafish somitogenesis oscillator. Curr. Biol. 13, 1398-1408 (2003).

22. Beilharz, T. H. et al. microRNA-mediated messenger RNA deadenylation contributes to translational repression in mammalian cells. PLoS One 4, e6783 (2009).

23. Bazzini, A. A., Lee, M. T. \& Giraldez, A. J. Ribosome profiling shows that mir-430 reduces translation before causing mRNA decay in zebrafish. Science 336, 233-237 (2012).

24. Zinovyev, A., Morozova, N., Gorban, A. N. \& Harel-Belan, A. Mathematical modeling of microRNA-mediated mechanisms of translation repression. In MicroRNA Cancer Regulation 189-224 (Springer, 2013).

25. Baek, J. H., Hatakeyama, J., Sakamoto, S., Ohtsuka, T. \& Kageyama, R. Persistent and high levels of Hesl expression regulate boundary formation in the developing central nervous system. Development 133, 2467-2476 (2006)

26. Gregor, T., Tank, D. W., Wieschaus, E. F. \& Bialek, W. Probing the limits to positional information. Cell 130, 153-164 (2007).

27. Hatakeyama, J. et al. Hes genes regulate size, shape and histogenesis of the nervous system by control of the timing of neural stem cell differentiation. Development 131, 5539-5550 (2004).

28. Karp, X. \& Ambros, V. Dauer larva quiescence alters the circuitry of microRNA pathways regulating cell fate progression in C. elegans. Development 139, 2177-2186 (2012).

29. Cheung, T. H. \& Rando, T. A. Molecular regulation of stem cell quiescence. Nat. Rev. Mol. Cell Biol. 14, 329-340 (2013).

30. Novák, B. \& Tyson, J. J. Design principles of biochemical oscillators. Nat. Rev. Mol. Cell Biol. 9, 981-991 (2008).

31. Cai, S., Zhou, P. \& Liu, Z. Functional characteristics of a double negative feedback loop mediated by microRNAs. Cogn. Neurodyn. 7, 417-429 (2013).

32. Huh, D. \& Paulsson, J. Non-genetic heterogeneity from stochastic partitioning at cell division. Nat. Genet. 43, 95-100 (2011).

33. Dunlap, J. C. Molecular bases for circadian clocks. Cell 96, 271-290 (1999).

34. Dequéant, M.-L. \& Pourquié, O. Segmental patterning of the vertebrate embryonic axis. Nat. Rev. Genet. 9, 370-382 (2008).

35. Shen, Q. et al. The timing of cortical neurogenesis is encoded within lineages of individual progenitor cells. Nat. Neurosci. 9, 743-751 (2006).

36. Okano, H. \& Temple, S. Cell types to order: temporal specification of CNS stem cells. Curr. Opin. Neurobiol. 19, 112-119 (2009). 
37. Bai, G. et al. ID sustains Hes1 expression to inhibit precocious neurogenesis by releasing negative autoregulation of Hes1. Dev. Cell 13, 283-297 (2007).

38. Balaskas, N. et al. Gene regulatory logic for reading the sonic hedgehog signaling gradient in the vertebrate neural tube. Cell 148, 273-284 (2012).

39. Zhao, C., Sun, G., Li, S. \& Shi, Y. A feedback regulatory loop involving microRNA-9 and nuclear receptor TLX in neural stem cell fate determination. Nat. Struct. Mol. Biol. 16, 365-371 (2009).

40. Paszek, P., Jackson, D. A. \& White, M. R. Oscillatory control of signalling molecules. Curr. Opin. Genet. Dev. 20, 670-676 (2010).

41. Kobayashi, T. et al. The cyclic gene Hes1 contributes to diverse differentiation responses of embryonic stem cells. Genes Dev. 16, 1870-1875 (2009).

42. Furusawa, C. \& Kaneko, K. A dynamical-systems view of stem cell biology. Science 338, 215-217 (2012).

43. Panovska-Griffiths, J., Page, K. M. \& Briscoe, J. A gene regulatory motif that generates oscillatory or multiway switch outputs. J. R. Soc. Interface 10, (2013).

44. Ding, X. C., Weiler, J. \& Grosshans, H. Regulating the regulators: mechanisms controlling the maturation of microRNAs. Trends Biotechnol. 27, 27-36 (2009).

45. Engelborghs, K., Luzyanina, T. \& Samaey, G. DDE-BIFTOOL v. 2.00: a Matlab package for numerical bifurcation analysis of delay differential equations, Report TW 330, Department of Computer Science. K.U. Leuven, Leuven, Belgium, 2001.

\section{Acknowledgements}

We thank Drs Shane Herbert, Peter Gould, Enrique Amaya and Matthew Ronshaugen for insightful comments on the manuscript, Professor Nick Monk for discussions and the Faculty of Life Sciences Systems Bioimaging Suite for access to appropriate microscopes and training. M.G., N.P., C.M. and this work were supported by a Wellcome Trust Senior
Research Fellowship award to N.P. (WT090868). N.E.P. is a PhD student in the Doctoral Training Centre for Systems Biology.

\section{Author contributions}

M.G. developed the computational model with input from N.P. M.G. performed the analysis. N.E.P. and T.G. contributed to the development and analysis of the model. C.M. performed the live cell imaging. N.P. supervised the work and co-wrote the manuscript with M.G.

\section{Additional information}

Supplementary Information accompanies this paper at http://www.nature.com/ naturecommunications

Competing financial interests: The authors declare no competing financial interests.

Reprints and permission information is available online at http://www.npg.nature.com/ reprintsandpermissions/

How to cite this article: Goodfellow, M. et al. microRNA input into a neural ultradian oscillator controls emergence and timing of alternative cell states. Nat. Commun. 5:3399 doi: 10.1038/ncomms4399 (2014).

\section{(c) (i)}

This work is licensed under a Creative Commons Attribution 3.0 Unported License. The images or other third party material in this article are included in the article's Creative Commons license, unless indicated otherwise in the credit line; if the material is not included under the Creative Commons license, users will need to obtain permission from the license holder to reproduce the material. To view a copy of this license, visit http://creativecommons.org/licenses/by/3.0/ 